\title{
Image Analysis and Synthesis Using Physics-Based- Modeling for Pearl Quality Evaluation System
}

\author{
Noriko Nagata', Toshimasa Dobashi ${ }^{2}$, Yoshitsugu Manabe ${ }^{2}$, Teruo Usami', \\ and Seiji Inokuchi ${ }^{2}$ \\ 'Industrial Electronics \& Systems Laboratory, Mitsubishi Electric Corporation, \\ 8-1-1, Tsukaguchi-honmachi, Amagasaki, 661 Japan \\ ${ }^{2}$ Department of Systems Engineering, Osaka University, \\ 1-3, Machikaneyama-cho, Toyonaka, 560 Japan
}

\begin{abstract}
Analysis by image synthesis using CG has attracted wide attention in machine vision. This paper proposes a method of modeling and synthesizing pearls that will be the central technique of a pearl quality evaluation system. Pearls manifest a specific optical phenomenon that is not dependent on the direction of the light source. To investigate this feature, we propose a physical model for multilayer film interference called an "illuminant model." The synthesis algorithm has been configured from such representations of physical characteristics as interference, mirroring and texture which correspond to the main evaluation factors obtained from human experts. Further, portions of photos of real pearls and the synthesized images were analyzed based on a scale of psychological evaluation of "pearl-like quality" demonstrating thereby that the generated images can present such a pearl-like quality.
\end{abstract}

\section{Introduction}

Image synthesis using computer graphics has recently come to be used in machine vision to enhance inspection systems. This approach is an analysis by synthesis method which is employed to find the optimum inspection conditions or inspection criteria through the simulation of the item for inspection. It is considered to be an important technology which will meet the needs to upgrade inspection systems and improving their accuracy.

In developing the pearl quality evaluation system, the authors have so far made various analytical approaches [1,2], and succeeded in deriving a relationship between the physical information regarding pearls and their evaluation by human experts. We have this time studied a synthetic approach which can form a virtual pearl sample in order to verify and correct the analytical results shown in Fig. 1.

Pearls are widely known and remarkably popular as jewelry items. They also have the specific optical and structural features given below [3].

- A pearl has a lustrous iridescence with its multilayer, thin-film structure, due to its diverse optical behavior such as interference and multiple reflection. Above all, the phenomenon of the hue distribution of the interference of light is characteristic.

- A pearl is a natural substance whose film thickness and surface roughness are nonuniform indicating natural irregularities and fluctuations.

The modeling and synthesis of a pearl, a substance interesting from the optical point of view as was seen above, is, therefore, a worthwhile topic for investigation.

Several studies have so far been made concerning the modeling of light behavior [4]. However little attention has been paid to multilayer, thin-film interference. Moreover, an investigation of the optical phenomenon of pearl mica paint has been reported [5], however, no such report has ever been made on the pearl. 


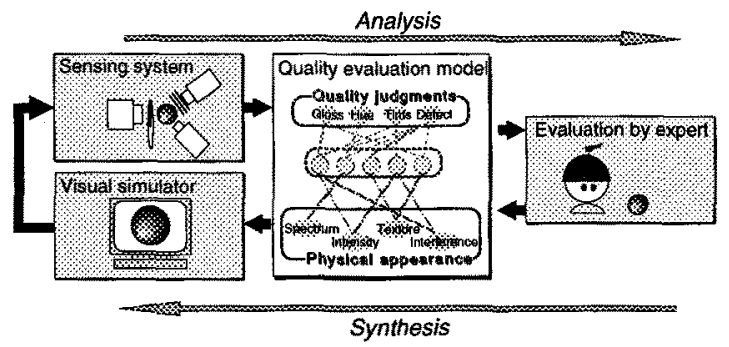

Fig. 1. Building up a pearl quality evaluation system by analytical and synthetic approach

The ultimate goal of this research is to clarify the inspection criteria by using various kinds of virtual samples, and collecting and analyzing the intuitive judgments of human experts. This paper proposes a pearl generating algorithm which will become one of the central techniques for the planned system. In order to represent the specific hue distribution, we propose a physical model called an "illuminant model," that deals with each point in the layer as a point light source. Also, it was revealed in our previous study that psychological factors in the quality evaluation of pearls include a sense of depth, of brightness and of grain [1]. Therefore, the image generating algorithm is configured from such representations of physical characteristics as interference, mirroring and texture, which correspond with these psychological factors. Furthermore, in the process of image synthesis a psychological scale we call the "pearl-like quality" is configured from portions of photos of real pearls, which are then matched with "pearl-like quality" compositions of the corresponding portions of the synthesized images in order to evaluate them.

\section{Modeling a Pearl}

\subsection{Physical Model of Multilayer Thin-Film Interference}

A pearl is composed of a nucleus and nacreous layers surrounding it. The nacreous layers are formed of translucent films of 300 to $800 \mathrm{~nm}$ thick aragonite crystallized layers and less than $20 \mathrm{~nm}$ thick protein membranes alternatively deposited concentricly in 1000 stacks. When the highly transparent crystallized layers are laminated uniformly, a lustrous iridescence appears due to interference and multiple reflection [3]. This phenomenon is regarded as a multilayer thin-film interference caused by 2 kinds of optical film [6].

The particular characteristic of the interference phenomenon of a pearl is the hue distribution of the interference color. Through the observation of a real pearl, we can see that the color appears on the opposite side to the light source where light does not hit directly, and changes concentrically from the center of the sphere. In other words, the interference color depends solely on the direction of the eyes, and not on the direction of the light source. In normal thin-film interference, the color change of the interference light largely depends on the direction of the light source. This is because the phase difference of two interference waves depends on the incident angle of the light source.

In order to simulate this phenomenon, we propose a physical model of multilayer thin-film interference called an "illuminant model" which pays careful attention to the multiple reflection of light inside a pearl as shown in Fig. 2. Some of the light reaching the pearl surface goes inside the pearl, is repeatedly reflected and transmitted and is 


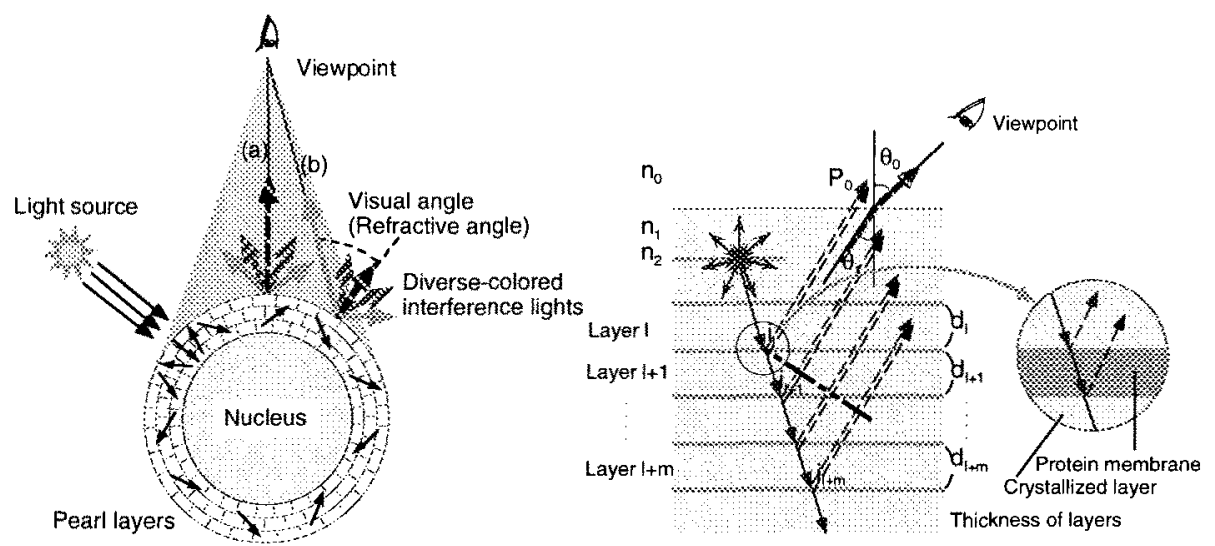

Fig. 2. Physical model of a pearl and interference of incident light in nacreous layer

propagated to the rear of the nucleus before being distributed over the whole nacreous layer. As a result, it appears as if each point in the layer had a point light source transmitting rays in all directions, with each ray causing local interference. Here, as the phase difference of the reflected wave is determined by the angle between the reflected wave and the nacreous layer, the power spectrum of the interference light depends only on the refractive angle. As the interference takes place everywhere in the nacreous layer, each interference ray is propagated in all directions outside the pearl. Taking account of only the interference light waves propagated in the direction of viewpoints ((a) and (b) in Fig. 2), the light from each point on the concentric circle is the interference light propagated with the same refractive angle, so that the phase difference, i.e. the spectrum distribution must be equivalent. It follows from this that the independence of the interference light color from the direction of the light source and its change in concentric form can thus be explained.

\subsection{Calculation Algorithm of Interference Light}

In Fig.2, the multilayer structure is composed of $L$ layers, each alternately composed of stacks of nacreous layers of thickness $d_{l}$ and of protein membranes. Here $n_{0}, n_{1}, n_{2}$ are the refractive indices of the air spaces, crystallized layers and protein membranes respectively.

First the layer film thickness column is generated, followed by casting a ray from the viewpoint to calculate the intersection with the pearl. The incident angle, reflectance and transmittance of all intersecting rays are calculated before making interference calculations from the outer layer to the inner layer of the nacreous layer for all visible wavelength bands in order to obtain the spectral power. The methods of calculation are given below.

Crystallized Layer Film Thickness Column. A film thickness generation method which imitates the growing process of a pearl can make it possible to represent non-uniformity of a natural pearl. As a method of generating the crystallized layer thickness column $d_{1}$, the thickness (400-700 nm) per layer and the number of films (1-3) per day are expressed by the normal distribution functions, and the thickness and number of films are further varied by using random numbers. The parameters of these functions are determined by the growth curve of the pearl [3]. 
Incident Angle. The light arriving at the viewpoint from point $P_{0}$ on the pearl surface is considered as follows: first, a single ray in a nacreous layer is reflected by the $l$ layer to the $l+m$ layer, and divided into the coherent rays $i_{1}$ to $i_{l+m}$, which later interfere with each other. Next, the interference light enters at $P_{0}$ from the nacreous layer with a refractive index $n_{1}$ to the air space of refractive index $n_{0}$ at the incident angle $\theta_{1}$, and refracts at the angle $\theta_{0}$. Therefore, from the viewpoint and the position of point $P_{0}$, the visual (refractive) angle $\theta_{0}$ is determined uniquely, and the incident angle $\theta_{1}$ is calculated from Snell's law.

Reflectance/Transmittance. The reflectance or transmittance is essentially determined by the refractive index of an object and the incident angle of light, and can be calculated using Fresnel's equations [6]. First, the energy reflectance $R_{1}$ ("reflectance") and the energy transmittance $T_{1}$ ("transmittance") when the light enters from the crystallized layer to the air space can be calculated by using Fresnel's equations in the following manner.

$$
\begin{aligned}
& R_{1}=\frac{1}{2}\left(|r p|^{2}+|r i|^{2}\right) \\
& T_{1}=1-R_{1}
\end{aligned}
$$

where: $r p=\frac{n_{1} \cos \theta_{0}-n_{0} \cos \theta_{1}}{n_{1} \cos \theta_{0}+n_{0} \cos \theta_{1}}, \quad r f=\frac{n_{1} \cos \theta_{1}-n_{0} \cos \theta_{0}}{n_{1} \cos \theta_{1}+n_{0} \cos \theta_{0}}$.

Here, $r p, r$ are the amplitude reflectances for p-polarized light and for s-polarized light respectively. The refractive index $n_{1}$ is calculated by taking the $\mathrm{C}$-axis refractive index of aragonite crystal as 1.53, and $n_{0}$ as 1.0. Next, the reflectance $R_{2}$ and the transmittance $T_{2}$ between the crystallized layer and the protein membrane are also calculated in the same manner.

Power Spectrum. Thinking of the light transmitted from the outer to the inner layer with the incident angle calculated above, the reflected waves are combined by calculating the phase differences between the reflected lights at the $l$ layer and the $l+k$ layer, which is computable for the wavelength $\lambda$ with the following formula.

$$
\xi=4 \pi \sum_{i=1+1}^{k} d\left(n_{1} / n_{0}\right) \cos \theta_{1} / \lambda
$$

The resultant waves are calculated by equation (3) and the reflectance and transmittance obtained above. These calculations are made for each visible wavelength band.

The interference calculation, starting from the 1st layer until the optical path difference reaches the coherence distance of natural light, is taken as one cycle of interference. Calculations are further made through the next layers until the light intensity fails to conform to the threshold value to obtain the spectral power.
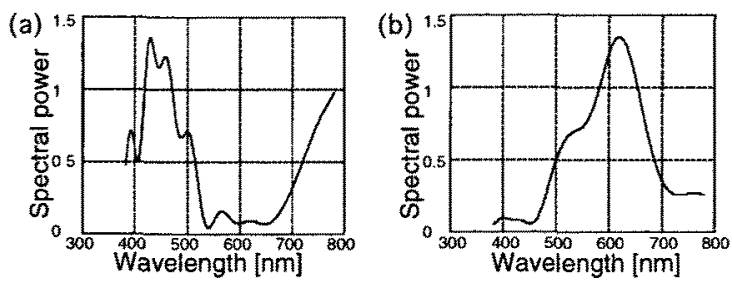

Fig. 3. Spectral power distribution of interference lights 
The interference light spectral power distribution at two given points is shown in Fig. 3 , indicating the flat spectrum distribution of white light being changed due to interference. Further, these correspond to the direction of viewpoint ((a) and (b) in Fig. 2) revealing that the spectrum distribution differs according to the visual angle.

\section{Synthesis of a Pearl Image}

The obtained interference light spectrum is converted into a RGB image, and is combined with components of specular reflection and diffuse reflection to synthesize the pearl image. In order to evaluate the pearl quality in particular, the three major psychological factors: sense of depth, sense of brightness and sense of grain, are given due weight.

\subsection{Representation of the Sense of Depth due to Interference Light}

The sense of depth corresponds to the expressions "thickly rolled," "strong tint," obtained through the questionnaires given to experts, and is considered to be related to the intensity of interference color [1]. Hence, the sense of depth is expressed by calculating the diffuse reflected light in a normal rendering model, and then varying the mixing ratio with the interference light. Fig. 4a shows the image of the diffuse reflected light. Fig. 4b shows an example of the image of the interference light component, where the bluish rainbow color, considered to be the most beautiful of the pearl interference lights, can be observed.

\subsection{Representation of the Sense of Brightness due to Mirroring}

Careful observation of a pearl shows that the background and the illumination of the circumference are mirrored well on the surface of the pearl. This was expressed by the experts as "mirroring one's face well [1]." In order to represent the difference in mirroring, the Torrance-Sparrow model is used for the light source, and the surface properties are expressed by varying the surface distribution and the decrement coefficient parameters. The ray tracing method is used to mirror the surrounding object, and the optical decrement effect due to the distance between the nacreous surface and the object is also provided. An example of the image of the mirrored light source and the table is given in Fig. 4c.

\subsection{Representation of the Sense of Grain due to Texture}

Unique textures expressed by "zara zara" (rough) or "mera mera" (flame like) are observed on the surface of a pearl. These textures are caused by the irregular striped patterns on the surface of a pearl. As a simple method of generating such textures, the high frequency component, extracted from the photograph of a real pearl by using two-dimensional FFT, band-passfilter and inverse FFT, is mapped on the nacreous surface. The example of texture (with expanded density scale) is shown in Fig. 4d.

\subsection{Example of Synthesis}

Examples of synthesis using this method are given in Fig. 4e. The diffuse image, interference component and mirroring component are combined. The interference color is calculated independently of the direction of the light source. However, a contradiction is not felt, and a realistic pearl interference can be represented. A sense of brightness and transparency is also expressed by mirroring. By varying the mixing ratio of the interference component and the diffuse image, the difference in the sense of depth is shown. In the 


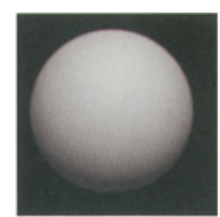

(a) An diffuse image

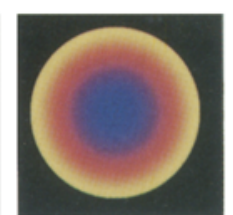

(b) An interference (c) component

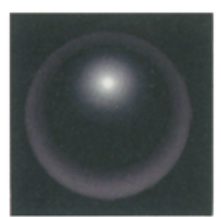

(c) A mirroring component

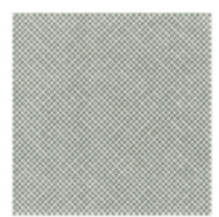

(d) Texture

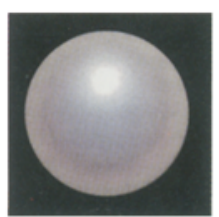

(f) A synthesized image with texture

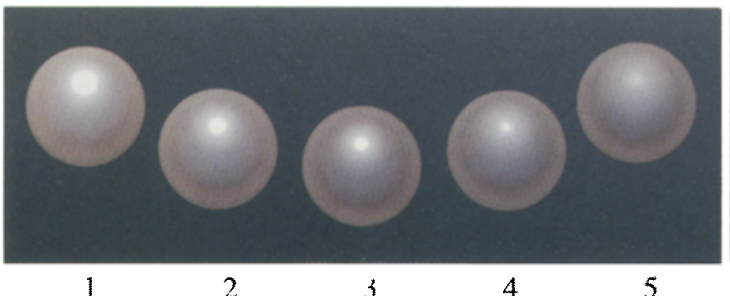

(e) Synthesized images

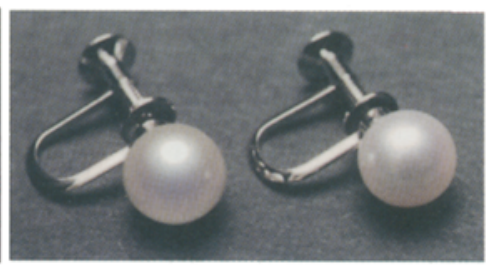

(g) A synthesized image (left) and a real pearl (right)

Fig. 4. Generated images

left three images (No. 1,2,3), the more to the right an image is the stronger is the sense of depth. By varying the parameters of the mirroring image, the difference in brightness is shown. In the right three images (No. 3,4,5), the left images are brighter. Figure 4 f shows the texture added to image No. 3 in Fig. 4e. Slight as the color change is, it is confirmed by experts that the change improves the sense of grain and reality on the surface of the pearl. To allow a comparison of our result with real pearls, the superimposition of the synthesized image on a photo of real pearls is shown in Fig. $4 \mathrm{~g}$. It follows, therefore, that this method can effectively represent the optical phenomena of pearls.

\section{Psychological Scaling of "Pearl-Like Quality"}

So far there is hardly any general method of making a quantitative evaluation of a $C G$ expressed image. In this section we would like to try the evaluation of synthesized images.

The CG representation method can be divided into two types-one is to bring the image infinitely close to the real object by using physical phenomenon (Expression of reality), and the other is to make the object more real than itself by effectively extracting (sometimes exaggerating) the features (Expression of abstraction). In consideration of the various restrictions such as calculation cost, the image synthesis can be carried out by making an abstract evaluation and by emphasizing the relevant factors, while utilizing a realistic expression, in order to bring the expression closer to the intrinsic essential quality.

Therefore, we closely examine this abstraction by using the key words "pearl-like quality". First, in order to learn what kind of spatial pattern a person senses in as a pearl, psychological experiments were carried out by using a photograph of a pearl in order to construct a psychological scale of pearl-like quality. Second, the synthesized images of a pearl are evaluated using the same method, and then compared with the photographs.

\subsection{Evaluation 1 Photo}

Seven kinds of subregion with different characteristics were cut from an enlarged 
photograph of a pearl as shown in Fig. 5. Next, 21 samples for evaluation, each consisting of two arbitrary subregions were made. A total of 103 university students were asked to make evaluations as to "which sample has the more pearl-like quality." For comparison, 2 rating cases were adopted: before and after observing a real pearl.

The psychological scale values due to the method of paired comparisons of Thurstone are shown in Fig. 6, with photographs 1 to 7 indicating the order from the center of a pearl. Further, the scale values obtained after and before showing the real pearl to men and women are respectively shown in Fig. 7. The following results were obtained.

- The result on the whole shows it was felt that the most pearl-like photograph was the one containing both specular reflections and interference colors (Photo 2). The photographs including the profiles were given poor ratings (Photos 5,6,7).

- The distance of psychological scale values between the groups of pearl-like photographs (Photos 1,2,3,4) and non pearl-like photographs (Photos 5,6,7) was found to be larger, comparing the dispersion in the groups, for the case when real pearls were observed than when they were not. Similarly, women, normally more familiar with pearls, apparently have a wider psychological scale distance than men. These values can evidently be taken as the scale for pearl-like quality.

These results thus indicate that a common psychological scale of pearl-like quality also exists among non-expert people. The sense of pearl-like quality depends on factors related to specular reflections and interference colors. It has also become clear that the profile of a pearl, i.e. the configurative factor is of little influence on the pearl-like quality.

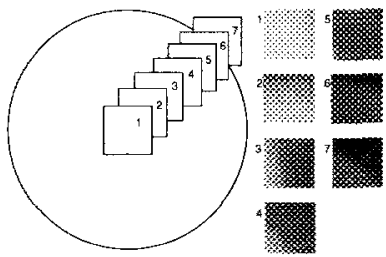

Fig. 5. Subregions of a picture of a pearl and their locations

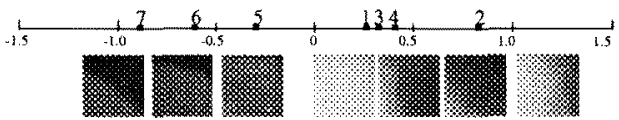

Fig. 6. A psychological scale of "pearl-like quality" - photograph -

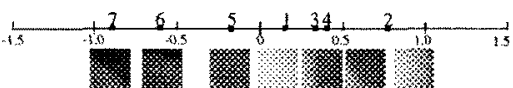

(a) Before observing a real pearl

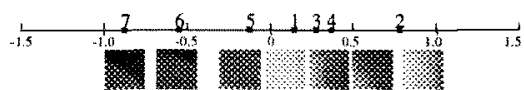

(c) Men

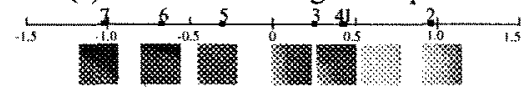

(b) After observing a real pearl

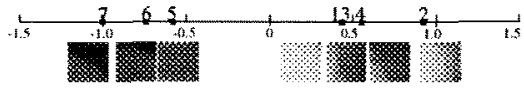

(d) Women

Fig. 7. Comparison of psychological scales

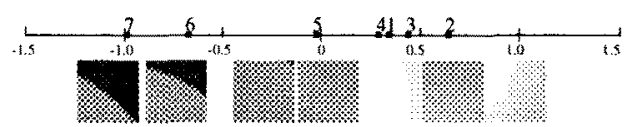

Fig. 8. A psychological scale of "pearl-like quality" - synthesized image - 


\subsection{Evaluation 2 synthesized image}

Seven kinds of subregion were cut from the synthesized image in the same manner as in Fig. 5 as samples for evaluation. A total of 50 university students were asked to evaluate the samples on the basis of their pearl-like quality.

The psychological scale values are given in Fig. 8. As compared with the order of scale values obtained from the photographs, the order is the same except for image No. 4 , which is 2 ranks down. No change in order is seen in the other samples, so that the synthesized images on the whole do give the features of the real pearl. The low ranking of image No. 4 and the value lowering of image No. 2 seem to be related to the smooth change in color and the roughness of the image.

It can, therefore, be deduced that the synthesized images can give not only entire but also partial representations of pearl-like quality. Also the pearl-like quality involves smoothness, particularly the smoothness in color change.

\section{Conclusion}

We have proposed a synthesis techniques for pearl images on the basis of a physical model and a psychological evaluation in building up an inspection system for pearls. This technique can be applied easily to analyze the inspection criteria of experts, which is the ultimate goal of our research. The parameters here are determined on the basis of experience, but the synthesis image has been found to be more than satisfactory for multilayer thin-film interference which has so far not been tried in the field of CG.

By using a psychological scale of pearl-like quality we have next evaluated the photographs of a real pearl and synthesized images. The results show that a synthesized image can make a partial as well as a total representation of the pearl-like quality. Furthermore, essential information has been acquired for representing real pearls. It is expected that the information can be used also in image generation to reduce the computing time by making a coarse calculation of factors not contributing to pearl-like quality.

However, the method needs further improvements. Some experts pointed out the lack of the sense of brightness in the synthesized images. In the representation of mirroring we would like to work on a physical model where the ooze of light are taken into account.

In the future we plan to study the correspondence of the psychological and physical factors of the inspectors on the basis of this model. We also plan to select the physical parameters that could contribute to the pearl-like quality.

\section{References}

1. Nagata, N., Kamei, M., Akane, M. and Nakajima, H.: Development of a pearl quality evaluation system based on an instrumentation of "Kansei". T. IEE Japan, 112-C, 2 (1992) 556-562

2. Nagata, N., Kamei, M. and Usami, T.: Transferring human sensibilities to machines - sensitivity analysis of layered neural networks and its application to pearl color evaluation -. Proc. IAPR Wks. on Machine Vision Applications (1994) 528-531

3. Wada, K. Pearl. The national jewelry association (1982)

4. Hanrahan, P. and Krueger, W.: Reflection from layered surfaces due to subsurface scattering. Computer Graphics Proc., Ann. Conf. Series (1993) 165-174

5. Gondek, J.S., Meyer, G.W. and Newman, J.G.: Wavelength dependent reflectance functions. Computer Graphics Proc., Ann. Conf. Series (1994) 213-220

6. Macleod, H.A.: Thin-film optical filters. Techno House (1986) 